\title{
Refining Image Search through Visual Similarities
}

\author{
Dipalee N. Gaikwad \\ Department of Computer Engineering \\ Dr. D.Y.Patil College of Engineering Ambi \\ Savitribai Phule Pune University
}

\author{
Sandeep U. Kadam \\ Department of Computer Engineering \\ Dr. D.Y.Patil College of Engineering Ambi \\ Savitribai Phule Pune University
}

\begin{abstract}
Retrieving the relevant images with respect to user query from a large image database is aim of the image search. So with respect to user query identifying the accurate image is the most challenging task. One of the important feature of multimedia is image retrieval. Some image search query results are satisfactory and some are unsatisfactory. To search over an image databases initially text based search approach is used where query text is matched with surrounding text of image. If surrounding information of an image is irrelevant then the search becomes inefficient. To improve the results of image search visual similarities are used. The similarity is checked between query image and images in the database. Images having higher similarity are retrieved, which makes search accurate.
\end{abstract}

\section{General Terms}

Metadata, Information Retrieval

\section{Keywords}

Image Retrieval, user intension, visual similarity, co-clicks, re-ranking.

\section{INTRODUCTION}

The important feature of multimedia is image search. In daily life this feature plays an important role. Image search results with respect to query image are sometimes satisfactory and sometimes unsatisfactory. Surrounding text of the image is considered for web image. Only by query keywords it is difficult to understand the intention of user and this leads to ambiguous image search results. Due to the success of information retrieval, most commercial search engines perform a text based search techniques for image search by using surrounding textual information. If the text information is sometimes noisy or unavailable, the drawback of such a retrieval method is that it cannot describe the contents of images precisely thus performance of image search is affected. To improve the performance of such image search, pattern matching can be used. With respect to user query to retrieve the relevant image from a large image database is the aim of the image search. So the most challenging task is to identify the accurate image with user intention. The traditional search engine takes query as input, if any image contains that query keyword in its metadata then that image is retrieved as result. If surrounding information of image is irrelevant and if that keyword is found into that information then this image is also displayed even it is not related with user intention. e.g. if user is searching for image of fruit "apple" then the image search results contains images of fruit apple, apple i-phone, apple laptop. In this proposed work Image re-ranking is based on similarities between query image and the group of images is performed. By observing click session information of user, which indicates visual similarities among the query images and group of images are compared. Images having high similarities with query image are retrieved. based on visual similarities of the images the search is refined. So the generated results are more efficient.

\section{LITERATURE SURVEY}

The traditional method of the image search includes only text based search. where the surrounding information of the image is compared with entered keywords. If the keywords are found then the image is displayed as resulting image [1]. To identify the intention of the user for which image he is searching for co-click history is used. Where the co-click defines the image A and image B are both clicked by user. If two images are coclicked frequently then they are more similar than third image co-clicked less often[3]. To express query of user in sufficient way keyword expansion is used. Which makes the query more descriptive[5].

\section{EXISTING SYSTEM}

Due to the success of text-based search of web pages, most search engines return images solely based on the text of the pages from which the images are linked. Text-based image search suffers from the ambiguity of query keywords. The keywords provided by users tend to be short. Also sometimes user gets difficulty in describing the visual content of target images using accurate keywords.

\section{PROPOSED SYSTEM}

The web image search plays an important role in daily life. User needs the accurate results for what he searches for. When user enters keyword for searching the image he has created some image in his mind that should be match with the resulting images. To generate accurate results of user query the proposed method performs the comparison between query image and group of images based on visual properties. Rather than searching with only meta information the search is refined with finding visual similarities. Co-click history is also used with visual similarities to refine the search and get accurate results[3]. The proposed system contains four modules. Which Accepts the query keyword as input based on that keyword the keyword expansion is done. User can also give image as input directly. Image is compared with other images in database the images having high similarity are displayed first. Fig 1 shows the block diagram of proposed system. Following are the proposed modules. 


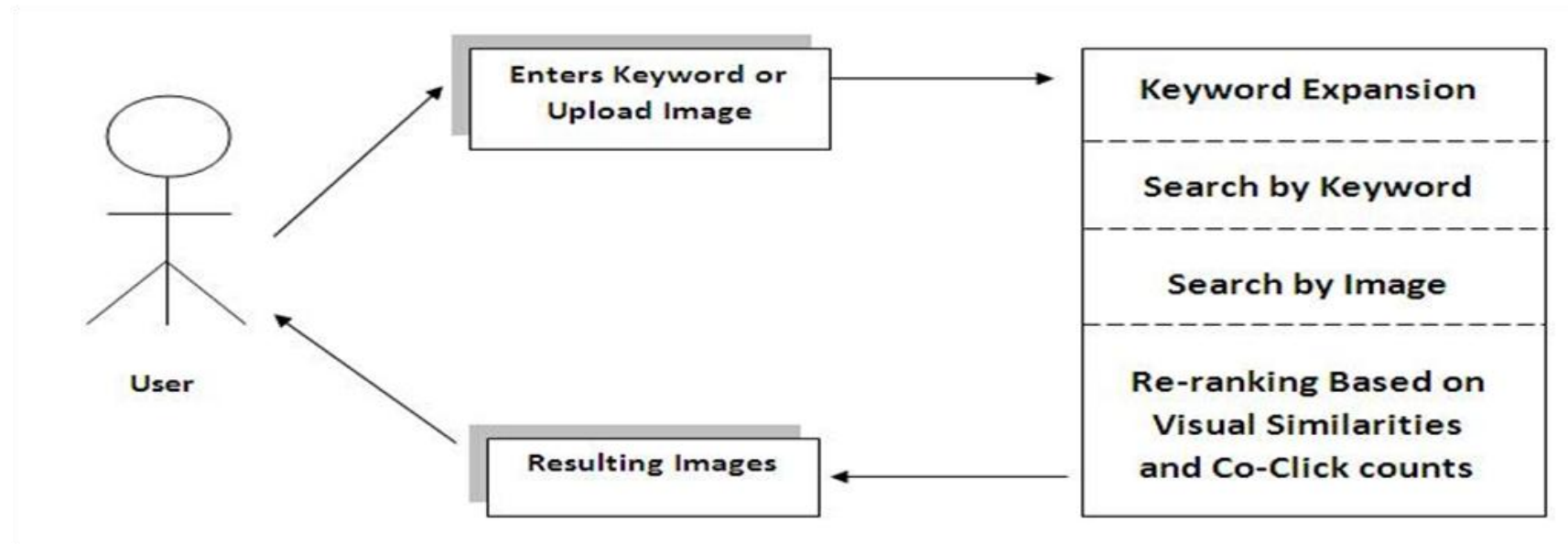

Fig. 1 Block Diagram of Proposed System

\subsection{Keyword Expansion}

User have to enter a keyword first for the image which he wants to search. When entering a keyword the keyword matching with already searched log history is suggested for expansion. The history also maintains the semantics of every keyword to make a search faster. The similar keywords with respect to user keyword are displayed first. From the suggested list User can select query keyword or user can enter new keyword. If user has query image and want to search similar images with the query image then he can upload the image to find similar image.

\subsection{Image Search with Query Keyword}

Based on the keyword entered by user the images are first searched from the database with their meta information. The meta information can be either file name, file information or any other text which describe an image. If the text matching with the entered keyword found in surrounding information of images then that images are retrieved as a result images. User can select one of the image from resulting images which are retrieved based on the keyword entered by user. User can give one of the image as query image to refinement the results. This Refinement is based on comparison of selected image with other images.

\subsection{Image Search with Query Image}

User can directly give image as input for which he want to search similar images. The query image is then compared with the images in database. Using the comparison algorithm the images are compared. The images having accuracy ratio greater than or equal to specified minimum accuracy are clustered and displayed. Following is the algorithm for image comparison.
Algorithm: To Compare images and to find the similarity between two images.

Input : Query Image

Output : List of appropriate match

Step 1: Split every pixel of image into three sub pixels red, green and blue.

Step 2 : Check color value of every pixel with respect to red, green ,blue.

Step 3 : Check color value of Query image against other database images.

Step 4: The final result is the average value of over all color values and all pixels.

Step 5: The calculated result is checked against an expected value.

Step 6: If the result is greater than expected value then images are said to be similar otherwise not.

\subsection{Re-ranking Based on Accuracy}

When user begins to search for the image a search session starts and it ends when the user exit from the search engine. Recording image click history of user clicks it is easy to find out co-relation between the images. During this search session user clicks multiple images from the set of images viewed. In a single image search session if image Qi and Qj are both clicked by the user then they are said as co-clicked. Two images that are co-clicked frequently are more similar to each other than to third image that is co-clicked less often. To find the image which is accurate with the user intension the 
comparison of co-click images are performed. $\Sigma \sigma\left(Q_{i}, Q_{j}, Q k\right) \begin{cases}Q_{j} & \text { if } \mathrm{C}(\mathrm{Xi}, \mathrm{Xj})>C(\mathrm{Xi}, \mathrm{Xk}) \\ \mathrm{Qk}_{\mathrm{k}} & \text { Otherwise }\end{cases}$

Where $\mathrm{C}(\mathrm{Qi}, \mathrm{Qj})$ is the number of search sessions where image Qi and Qj are co-clicked. Images with given query results are Re-ranked according their co-click history. By maintaining past history of co-clicks is also helpful for faster results.

\subsection{Re-ranking Based on Accuracy}

Images having accuracy ratio greater than specified minimum ratio are taken to display the result. These images are reranked based on their co-click counts. The image having coclick count greater is displayed first. So by considering coclick count the images are re-ranked

\section{WORKING OF SYSTEM WITH}

\section{RESULTS}

In proposed system user can give query keyword as input or user can directly give image as input to find out the related images. Following is some of the execution of proposed work

\subsection{Search by Keyword}

Fig. 2 shows the search performed using keyword apple. The results of this search includes all the images including meta information as apple. The meta information considered is filename, surrounding information of the image. If the query keyword is found into metainformation then that images are displayed to the user as resulting image. User have to select one of the image from the resulting images. Selected image is compared with other images with their visual similarities. By taking into consideration the color values that are R, G, B. When user enters keyword the image of apple i phone is also displayed as it contains the word apple into its meta information. User can select one of the image from the resulting image.

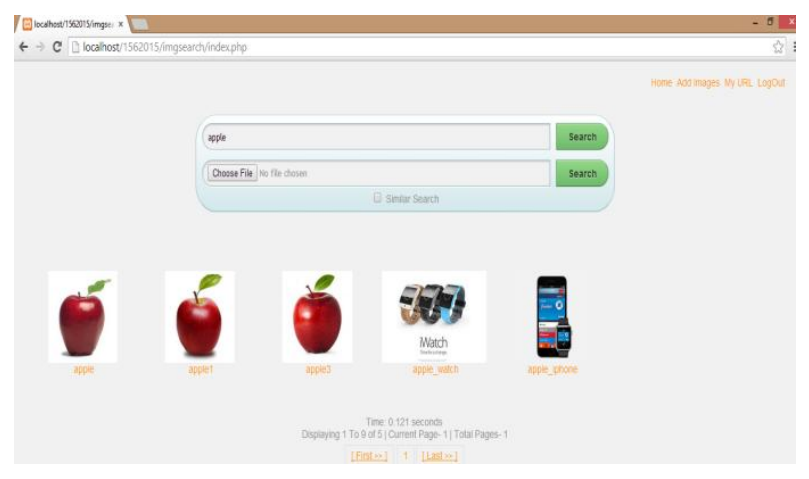

Fig. 2 Search by Keyword

Fig 3. Shows the graph of keyword based image search. This retrieval of the image is with respect to query keyword. The graph shows at what time which image is retrieved. $\mathrm{X}$ axis indicate the image names where as the $\mathrm{Y}$ axis indicate the time in milliseconds.

\subsection{Search by Image}

After selecting one of the image from the resulting image or either giving any image as input that image is compared with

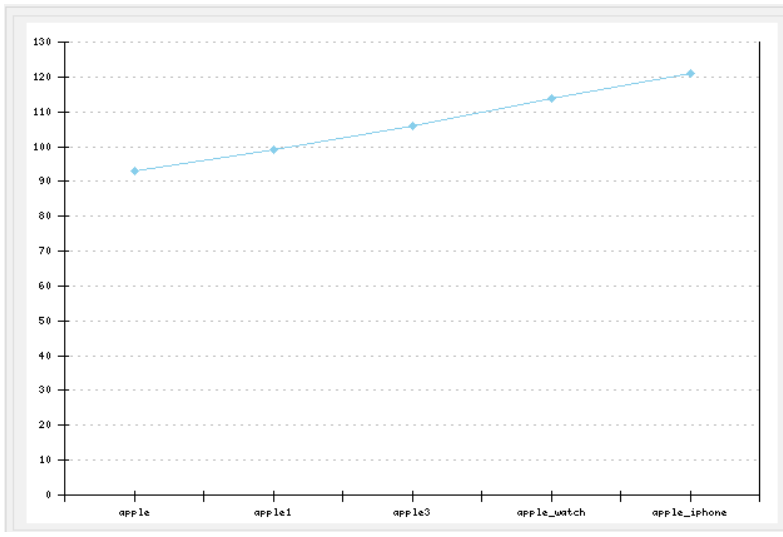

Fig. 3 Graph of keyword based Search

other images. In Fig. 4 fruit apple is chosen as query image and search is performed. The results are displayed in Fig. 4. Which contains all the images similar to query image. The images displayed are based on features(Visual) similar to the query image. This shows the refinement of the results which contains all the images related with query image

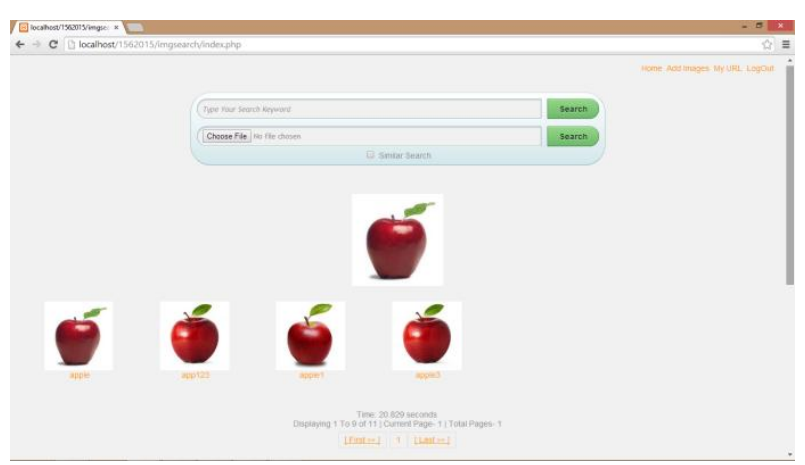

Fig. 4 Search by Image

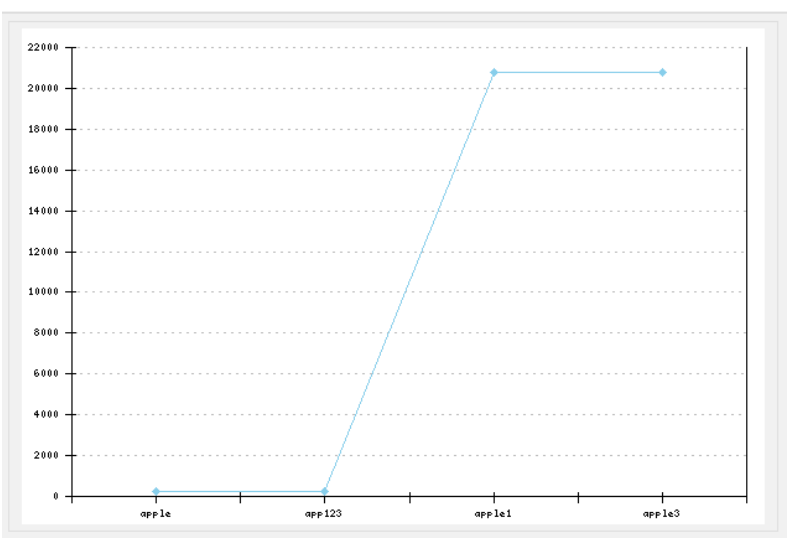

Fig. 5 Graph of Image based Search 
Fig. 5 shows the graph of image based search. This retrieval of the image is with respect to Image. The graph shows at what time which image is retrieved. $\mathrm{X}$ axis indicate the image names where as the $\mathrm{Y}$ axis indicate the time in milliseconds.

For e.g. the user is searching for fruit apple, water glass, computer mouse and cricket bat. Following table shows details for search by keyword. The column indicates

\section{A= Given input keyword}

$\mathrm{B}=$ Number of images containing given keyword as metainformation

$\mathrm{C}=$ Number of images in database which matches actual user intention

$D=$ Number of images retrieved as result for search by keyword

$\mathrm{E}=$ Accurracy $=(\mathrm{D} / \mathrm{C}) * 100$

$\mathrm{F}=$ Given Query Image

$\mathrm{G}=$ No of images retrieved as result for search by image

$\mathrm{H}=$ Number of images retrieved as result for search by image

$\mathrm{I}=\operatorname{Accuracy}(\mathrm{H} / \mathrm{C}) * 10$

Table 1. Accuracy for Search by keyword

\begin{tabular}{|l|l|l|l|l|}
\hline A & B & C & D & E \\
\hline apple & 12 & 10 & 5 & 50 \\
\hline glass & 14 & 10 & 4 & 40 \\
\hline mouse & 15 & 10 & 5 & 50 \\
\hline bat & 10 & 5 & 3 & 60 \\
\hline
\end{tabular}

Table 2. Accuracy for Search by image

\begin{tabular}{|l|l|l|l|}
\hline F & C & H & I \\
\hline Fruit apple & 10 & 10 & 100 \\
\hline Water glass & 10 & 9 & 90 \\
\hline Computer mouse & 10 & 9 & 90 \\
\hline Cricket bat & 5 & 5 & 50 \\
\hline
\end{tabular}

Fig. 6 shows the comparison between search by keyword and search by image based on values in table 1 and table 2 . Search by image method is more accurate than search by keyword. Fig. 6 shows searching of four different images where $\mathrm{X}$ axis indicates user intension and $\mathrm{Y}$ axis indicates Accuracy of results returned by search by keyword and search by image.

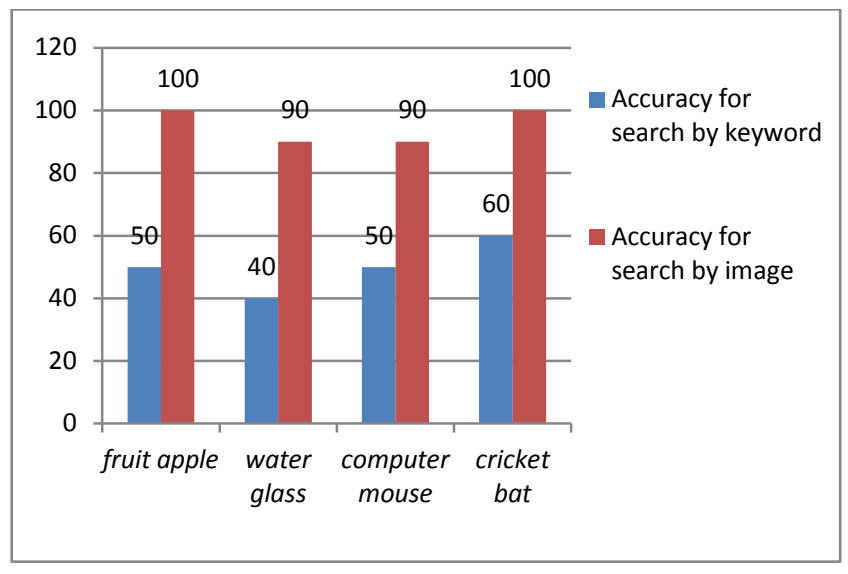

Fig 6. Comparison of search by keyword and search by image

\subsection{Search by Keyword and Image}

The following Fig. 8 shows Combination of search by keyword and search by image approach. Keyword and image both are given as input. Here the keyword given is apple and image given is the image of fruit apple. For comparison it takes into consideration only images which include image name or metainformation as apple. Visual similarity is checked for only images having metainformation as apple and based on click count re-ranked images are displayed.

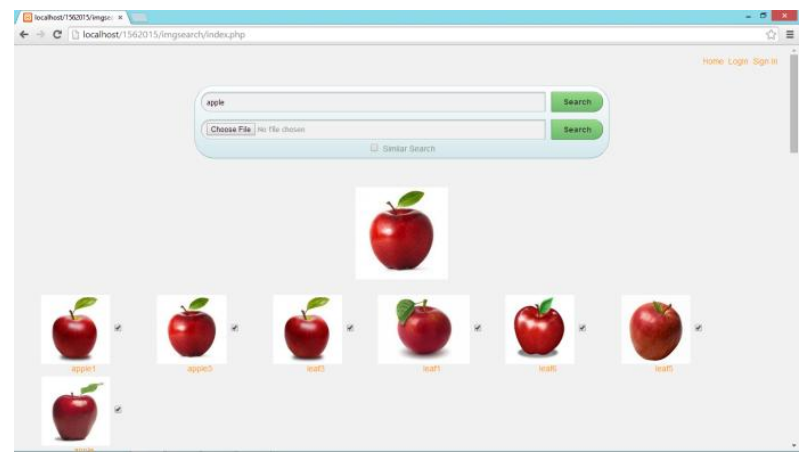

Fig. 7 Search by keyword and Image

Using combination of Search by keyword and search by image is the images having name apple are compared with query image for visual similarity.

\section{CONCLUSION}

To remove unreliability and to increase the performance of web search engine multiple methods are applied. In this proposed work Image search performed is based on similarities between query image and the group of images. By observing click session information of user, which indicates visual similarities among the query images and group of images are compared. Images having high similarities with query image are retrieved. The search is refined based on visual similarities of the images. So the generated results are more efficient. By using keyword expansion most relevant 
images with query image are found. Co-click session derived from text based search engine query logs provides fastest results for similarity based search. Overall performance of the search engine is improved using visual similarities between query image and pool of the images

Image Search can be extended to minimize information leak and preserving data confidentiality against unauthorized access provided for web image search.

\section{REFERENCES}

[1] Yushi Jing, Michele Covell, David Tsai, and James M. Rehg, Member, IEEE, Learning Query-Specific Distance Functions for Large-Scale Web Image Search, IEEE Transactions On Multimedia, Vol. 15, No. 8, December 2013.

[2] Zheng Lu, Xiaokang Yang, Senior Member, IEEE, Weiyao Lin, Hongyuan Zha, and Xiaolin Chen, Inferring User. Image-Search Goals Under theImplicit Guidance of Users, IEEE Transactions On Circuits And Systems For Video Technology, Vol. 24, NO. 3, MARCH 2014.

[3] R.Datta, D.Joshi, J.Li, and J.Z.Wang, “Image retrieval:Ideas,influences, and trends of the new age", ACM Comput. Surveys, vol. 40, no. 2, pp. 1-5, 2008

[4] Xiaogang Wang, Member, IEEE, Shi Qiu, Ke Liu, and Xiaoou Tang, Fellow, IEEE, Web Image Re-Ranking, Using Query-Specific Seman- tic Signatures, IEEE Transactions On Pattern Analysis And Machine Intelligence, Vol. 36, No. 4, April 2014

[5] Yogdong Zhang, Senior Member, IEEE, Xiaopeng Yang, and Tao Mei, Senior Member, IEEE, Image Search Re- ranking with Query-dependent Click-based Relevance Feedback, IEEE TRANSACTIONS ON IMAGE PROCESSING, 1057-7149 (c) 2013 IEEE.

[6] Xiaoou Tang, Fellow, IEEE, Ke Liu, Jingyu Cui, Student Member, IEEE, Fang Wen, Member, IEEE, and xiaogang Wang, Member, IEEE, IntentSearch: Capturing User Intention for One-Click Internet Image Search , IEEE Transactions On Pattern Analysis And Machine Intelli- gence, Vol. 34, NO. 7, JULY 2012.

[7] Xinmei Tian, Dacheng Tao, Member, IEEE, Xian-Sheng Hua, Member, IEEE, and Xiuqing Wu, Active Reranking For Web Image Search, IEEE Transactions On Image Processing, Vol. 19, No. 3, March 2010.

[8] Linjun Yang, Member, IEEE, and Alan Hanjalic, Senior Member, IEEE, Prototype-Based Image Search Reranking, IEEE Transactions On Multimedia, Vol. 14, No. 3, June 2012871

[9] Xinmei Tian, Yijuan Lu, Member, IEEE, and Linjun Yang, Member, IEEE, Query Difficulty Prediction for Web Image Search, Transactions On Multimedia,Vol. 14,NO. 4, 2012951

[10] Shikui Wei, Dong Xu, Xuelong Li, Fellow, IEEE, and Yao Zhao, Senior Member, IEEE, "Joint Optimization. Toward Effective and Efficient Image Search" IEEE Transactions On Cybernetics, Vol. 43, No. 6 December 2013

[11] Dipalee Gaikwad, Sandeep U. Kadam, "Survey on Methodologies Used for Web Image Search", International Journal of Computer Applications(09758887), Volume 107- No.18, December 2014 